\title{
Adult patients with respiratory syncytial virus infection: impact of solid organ and hematopoietic stem cell transplantation on outcomes
}

\author{
P. Pilie, W.A. Werbel, J. Riddell IV, X. Shu, D. Schaubel, K.S. Gregg. \\ Adult patients with respiratory syncytial virus infection: impact \\ of solid organ and hematopoietic stem cell transplantation on \\ outcomes. \\ Transpl Infect Dis 2015: 17: 551-557. All rights reserved \\ Abstract: Background. Respiratory syncytial virus (RSV) is a \\ common community-acquired pathogen responsible for a substantial \\ disease burden in adults. We investigated the outcomes after RSV \\ infection in hospitalized adults over a 3-year period. \\ Methods. This single-center, retrospective study identified 174 \\ patients hospitalized with RSV upper or lower respiratory tract \\ infection (LRTI) between January 1, 2009 and June 30, 2012. Clinical \\ data were extracted from medical records. The primary outcome \\ analyzed was all-cause mortality, defined as death during the index \\ hospital admission. Subjects were divided into 3 groups for \\ comparison: hematopoietic stem cell transplant (HSCT) patients, \\ solid organ transplant (SOT) patients, and non-transplant patients. \\ Results. In our study, 41/174 (23.6\%) were HSCT recipients and 28/ \\ $174(16.1 \%)$ were SOT recipients. Twelve of $174(6.9 \%)$ died. Death \\ occurred in 2/41 (4.9\%) HSCT and 3/28 (10.7\%) SOT recipients, \\ compared to $7 / 106$ (6.6\%) non-transplant patients. When compared \\ to the non-transplant cohort, HSCT and SOT were not found to be \\ significant risk factors for mortality $(P=0.685$ and 0.645 , \\ respectively). In multivariate logistic regression, age $>60$ was \\ associated with mortality $(P=0.019)$, while lymphopenia on \\ admission trended toward an association with death $(P=0.054)$. \\ HSCT patients were less likely to be admitted to an intensive care \\ unit (odds ratio [OR] $0.26, P=0.04$ ), but were significantly more \\ likely to receive ribavirin therapy (OR 11.62, $P<0.0001$ ). \\ Conclusion. Adults hospitalized with RSV LRTI are at significant risk \\ of mortality, and this risk may be increased in patients age $>60$ or \\ with lymphopenia on admission. This study did not identify any \\ significant increased mortality or morbidity associated with RSV \\ infection in immune suppressed transplant recipients vs. patients \\ who had not received a transplant.
}

\author{
P. Pilie', W.A. Werbel ${ }^{2}$, J. Riddell IV ${ }^{3}$, \\ X. Shu ${ }^{4}$, D. Schaubel ${ }^{4}$, K.S. Gregg ${ }^{3}$ \\ ${ }^{1}$ Department of Internal Medicine, University of Michigan, \\ Ann Arbor, Michigan, USA, ${ }^{2}$ Department of Internal \\ Medicine, Northwestern University Feinberg School of \\ Medicine, Chicago, Illinois, USA, ${ }^{3}$ Division of Infectious \\ Diseases, University of Michigan Medical School, Ann \\ Arbor, Michigan, USA, ${ }^{4}$ Department of Biostatistics, \\ School of Public Health, University of Michigan, Ann \\ Arbor, Michigan, USA
}

Received 2 December 2014, revised 9 April 2015 , 8 May 2015, accepted for publication 21 May 2015

\section{DOl: $10.1111 /$ tid. 12409}

Transpl Infect Dis 2015: 17: 551-557
Respiratory syncytial virus (RSV) is a single-stranded, enveloped RNA virus of the paramyxoviridae family that causes respiratory infection in 3-20\% of adults annually, rivaling the incidence of influenza $\mathrm{A}$ infection (1-6).

Multiple studies have identified risk factors for severe RSV infection in adults including advanced age, poor functional status, underlying cardiopulmonary disease, and significant immunocompromised state $(7,8)$. Hematopoietic stem cell transplant (HSCT) recipients represent a particularly high-risk group, with recent studies showing significant but highly variable mortality rates of $5-80 \%$ in patients with lower respiratory tract infection (LRTI) (9-16). In comparison, 
studies involving solid organ transplant (SOT) recipients have only demonstrated significant mortality in lung transplant recipients with LRTI (0-20\%), although published data for other organs are limited $(14,17,18)$. However, measured outcomes of transplant recipients infected with RSV in prior studies are heterogeneous, as are treatment approaches. Unlabeled use of ribavirin, a guanosine analog, has been used as a primary therapy for RSV; however, owing to a lack of large randomized control trials, currently there is no standardized treatment strategy in these at-risk populations. Therefore, in this study, we present a retrospective analysis of 174 RSV assay-confirmed cases, including both transplanted and non-transplanted adults, between 2009 and 2012 at the University of Michigan Hospital, designed to better characterize risk factors leading to severe infection and death, as well as outcomes of RSV LRTI in a diverse hospitalized population.

\section{Patients and methods}

This study was conducted at the University of Michigan Hospital, a 980-bed tertiary care medical center, where approximately 350 SOTs and 250 HSCTs are performed each year. After institutional review board approval, subjects were identified using retrospective data of positive RSV viral culture, antigen assay (Diagnostic Hybrids, Athens, Ohio, USA), or polymerase chain reaction (PCR) assay (Prodesse ProFlu+; Hologic, Bedford, Massachusetts, USA) (19) obtained between January 1, 2009 and June 30, 2012, encompassing 4 sequential RSV seasons. In total, 218 adult patients were identified; 44 of these patients were diagnosed and managed as outpatients, and were therefore excluded from analysis, leaving 174 hospitalized subjects for inclusion.

Data related to the hospitalization and outcomes of each patient were abstracted from the electronic medical record system. Demographic data included age, gender, history of malignancy or chronic lung disease (such as chronic obstructive pulmonary disease, interstitial lung disease, pulmonary fibrosis, or bronchiectasis), ongoing chemotherapy, receipt of autologous or allogeneic HSCT, and receipt of SOT. Clinical data abstracted from the medical record included interval time from HSCT or SOT to diagnosis of RSV infection, presenting symptoms, and oxygen saturation at time of admission, chest radiographic findings (x-ray and computed tomography), admission to intensive care unit during hospitalization, mechanical ventilation, pulmonary co-infection(s), receipt and duration of oral and/or inhaled ribavirin, and duration of hospitalization. Subjects were determined to have had upper respiratory tract infection (URTI) or LRTI based on these data. LRTI was present if either of the following criteria were met: (i) pulse oximetry $<90 \%$ or requirement of supplemental oxygen therapy on admission with respiratory symptom(s) of infection and radiographic evidence of LRTI and positive nasopharyngeal or throat RSV assay, or (ii) bronchoalveolar lavage specimen positive for RSV. Any subject not meeting LRTI criteria but with an upper respiratory sample positive for RSV and concurrent respiratory tract infection symptoms was defined as having URTI. All testing for RSV at our hospital during the study period was performed at the discretion of the treating physician(s).

Data were obtained from the date of hospital admission until the date of discharge. The primary outcome analyzed was all-cause mortality, defined as death during the index hospital admission. To study the predictors of death (covariate-adjusted), a logistic regression model was used. Covariates included in the model were age, gender, absolute lymphocyte count (ALC) on admission, transplant status, diagnosis of chronic lung disease, fever, computed tomography findings, and URTI symptoms. Age at diagnosis was split into 2 categories: $>60$ years and $\leq 60$ years.

Secondary analysis was performed with subjects split into SOT, HSCT, and non-transplanted cohorts. Multivariate logistic regression models for death were fitted separately in these 3 groups. Because of the small sample size, some covariates did not have enough variation and, thus, were excluded from the model. The standard significance level (0.05) was applied to delineate statistically significant from non-significant effects.

In addition, in order to describe observed patterns in the number of RSV diagnoses over calendar time, curves displaying RSV incidence (counts of diagnoses vs. time) were plotted by transplant type. All statistical analyses were carried out using SAS v9.2 (SAS Institute, Cary, North Carolina, USA).

\section{Results}

During the study period, 174 cases of RSV infection occurred in hospitalized adult patients. Of these, 152 cases $(87.3 \%)$ were identified by positive nasopharyngeal swab (150 PCR assay, 2 viral culture); 13 cases (7.5\%) identified by bronchoalveolar lavage fluid (10 PCR assay, 3 viral culture); and 9 cases (5.2\%) identified by sputum culture/tracheal aspirate (4 PCR assay, 3 RSV antigen, 2 viral culture). Epidemiologic curves by transplant type are shown in Figure 1 and include both 


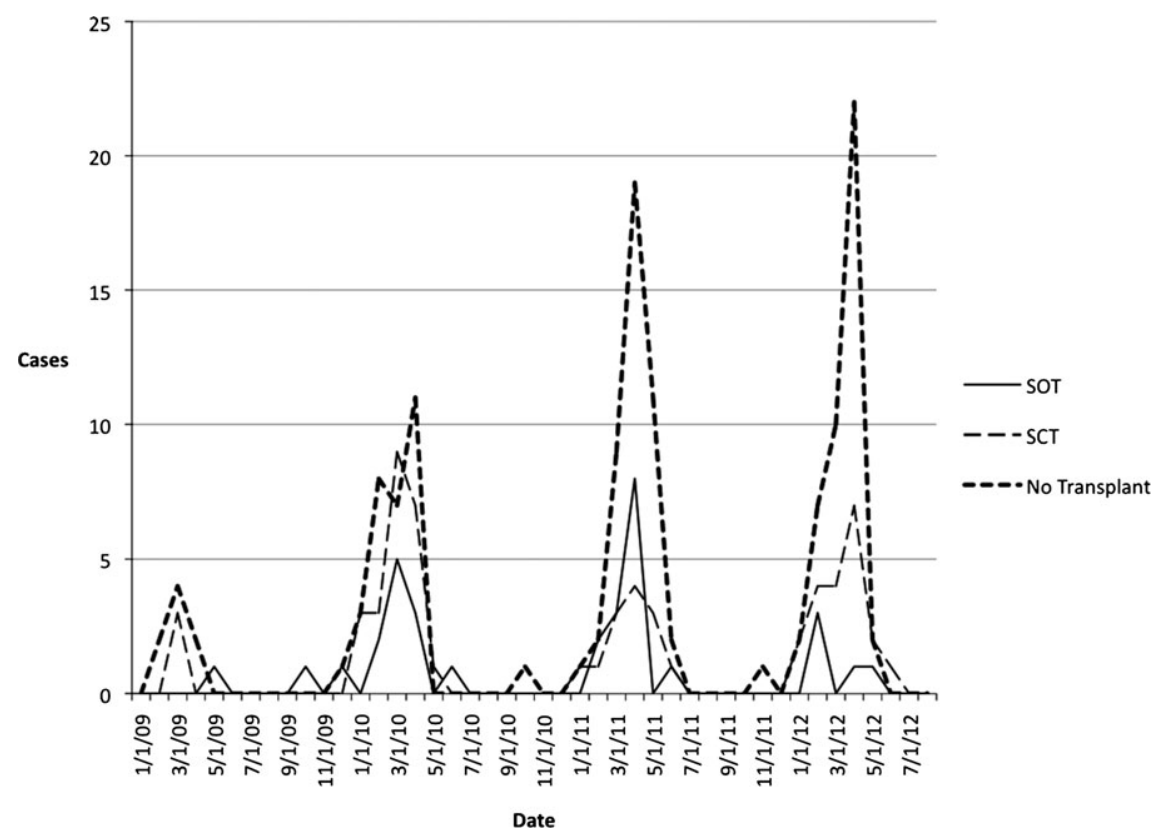

Fig. 1. Epidemiologic curve of confirmed cases of respiratory syncytial virus infection by transplant type: 2009-2012. SOT, solid organ transplant; SCT, stem cell transplant.

outpatient and inpatient subjects. Fifteen RSV cases were detected in the 2009 season, 64 cases in 20092010, 72 cases in 2010-2011, and 67 cases in 2011-2012. Of note, the University of Michigan microbiology laboratory transitioned to PCR-based testing for RSV in September 2009 and, thereafter, a consistently higher number of cases were identified each season. The periodic pattern of the curves and the time of onset of infection among SOT, HSCT, and non-transplant cohorts were similar in each season, with cases primarily identified between January and May.

Patient characteristics of the 174 subjects are listed in Table 1 . Of these, 41 subjects (23\%) were HSCT recipients, 28 subjects (16\%) were SOT recipients, and 106 subjects $(61 \%)$ were not transplant recipients. The SOT cohort included 16 lung, 8 kidney, 3 heart, 1 liver, and 1 kidney/pancreas transplant recipient. One patient received both a HSCT and SOT. LRTI was present in 121 subjects (69.5\%). The average length of stay at the hospital was $9.3 \pm 11.3$ days; 37 subjects (21.2\%) required intensive care unit admission, and 17 (9.8\%) received mechanical ventilation. The non-transplant cohort was significantly older than the HSCT cohort (62.1 years vs. 53.8 years, $P=0.001$ ). Subjects with SOT and non-transplanted subjects were both significantly more likely to have underlying chronic lung disease than were HSCT subjects $(P=0.003)$. The mean durations from date of HSCT or SOT until
RSV infection were 2.60 years and 6.05 years, respectively.

Twelve subjects (6.9\%) died during hospitalization. 2 subjects were HSCT recipients, 3 were SOT recipients, and 7 were not transplant recipients. All deaths occurred in patients with LRTI, resulting in a mortality rate of $10 \%(12 / 121)$ in this subset of patients. Results for the logistic regression model of death are listed in Table 2. Death risk was significantly greater for subjects $>60$ years of age, with a covariate-adjusted odds ratio $(\mathrm{OR})$ of $8.41(P=0.019)$ compared with subjects $<60$ years of age. Each 1 unit $(100$ cells $/ \mu \mathrm{L})$ increase in ALC on admission was estimated to decrease the odds of in-hospital death by approximately $75 \%(\mathrm{OR}=0.24)$, with the effect approaching statistical significance $(P=0.051)$. No other covariate effects approached significance for risk of death.

Results based on logistic regression models of death in HSCT recipients $(n=41)$ and SOT recipients $(n=28)$ did not identify any covariates associated with mortality. For non-transplant subjects, higher ALC was significantly associated with lower odds of death $(\mathrm{OR}=0.09 ; P=0.046)$. Specifically, each 100 cell $/ \mu \mathrm{L}$ increase in ALC resulted in a $>90 \%$ decrease in the risk of death. Age $>60$ showed borderline significance, with a trend toward greater risk of mortality $(\mathrm{OR}=11.4 ; P=0.065)$; i.e., the odds of death in patients age $>60$ was more than 11 times greater than 
Baseline characteristics of study patients

\begin{tabular}{|c|c|c|c|}
\hline Characteristics & $\begin{array}{l}\text { Inpatients with SCT } \\
(n=41)^{1}\end{array}$ & $\begin{array}{l}\text { Inpatients with SOT } \\
(n=28)^{1}\end{array}$ & $\begin{array}{l}\text { Inpatients without } \\
\text { transplant }(n=106)\end{array}$ \\
\hline \multicolumn{4}{|l|}{ Gender, $n(\%)$} \\
\hline Female & $16(39.02)$ & $10(35.71)$ & 75 (70.75) \\
\hline Male & $25(60.98)$ & $18(64.29)$ & $31(29.25)$ \\
\hline Age, mean years \pm SD & $53.78 \pm 11.82$ & $55.00 \pm 15.12$ & $62.08 \pm 19.83$ \\
\hline Chronic lung disease, $n(\%)$ & $4(9.76)$ & $11(39.29)$ & 36 (33.96) \\
\hline Length of stay, mean days \pm SD & $11.44 \pm 13.51$ & $9.29 \pm 11.51$ & $8.49 \pm 10.30$ \\
\hline ICU admission, $n(\%)$ & $6(14.63)$ & $5(17.86)$ & $26(24.53)$ \\
\hline Mechanical ventilation, $n(\%)$ & $3(7.32)$ & $4(14.29)$ & $10(9.43)$ \\
\hline Death, $n(\%)$ & $2(4.88)$ & $3(10.71)$ & $7(6.60)$ \\
\hline Fever, $n(\%)$ & $22(53.66)$ & $13(46.43)$ & $45(42.45)$ \\
\hline \multicolumn{4}{|l|}{ Respiratory infection, $n(\%)$} \\
\hline Upper & $11(26.83)$ & $10(35.71)$ & 32 (30.19) \\
\hline Lower & $30(73.17)$ & $18(64.29)$ & $74(69.81)$ \\
\hline Lymphocyte count ${ }^{2}$, mean \pm SD & $1234 \pm 831$ & $860 \pm 528$ & $1208 \pm 1059$ \\
\hline \multicolumn{4}{|l|}{ Abnormal CT findings, $n(\%)$} \\
\hline Yes & $19(46.34)$ & $8(28.57)$ & $23(21.70)$ \\
\hline No & $7(17.07)$ & $5(17.86)$ & $7(6.60)$ \\
\hline CT scan not performed & $15(36.59)$ & $15(53.57)$ & $76(71.70)$ \\
\hline Treated with ribavirin, $n(\%)$ & $23(56.10)$ & $6(21.43)$ & $10(9.43)$ \\
\hline $\begin{array}{l}\text { Duration from transplant to } \\
\text { infection, mean years } \pm S D\end{array}$ & $2.60 \pm 2.47$ & $6.05 \pm 6.69$ & NA \\
\hline \multicolumn{4}{|c|}{$\begin{array}{l}{ }^{1} \text { One subject had both SCT and SOT, and thus was counted in both groups. } \\
{ }^{2} \text { Lymphocyte count data not available for } 5 \text { subjects. }\end{array}$} \\
\hline
\end{tabular}

Table 1

that of patients age $\leq 60$, adjusting for all other covariates.

Ribavirin therapy (either inhaled or systemic) was administered in 39 (22.4\%) patients. Overall, 23/40 (56.1\%) of HSCT recipients received some form of ribavirin therapy, compared with $6 / 27(22.2 \%)$ of SOT recipients, and 10/107 (9.3\%) of non-transplant recipients. In those who received ribavirin therapy, LRTI was identified in $16 / 22(72.7 \%)$ HSCT recipients, in 5/6 (83.3\%) SOT recipients, and in 8/10 (80\%) non-transplant recipients. The average duration of ribavirin therapy was 7.8 days (range 1-15 days, median 7 days). In ribavirin-treated patients, 5/39 (12.8\%) received both inhaled and oral ribavirin, all 5 of whom were HSCT recipients, with 1 in-hospital death. Thirtyfour of 39 patients $(87.2 \%)$ received only oral ribavirin (18 HSCT recipients, 6 SOT recipients, and 10 non-transplanted subjects), with 3 in-hospital deaths (1 HSCT recipient, 1 SOT recipient, 1 non-transplant).

\section{Discussion}

RSV has been shown to be an important cause of respiratory infection in transplant recipients with significant associated morbidity and mortality. In this single-center study, we retrospectively analyzed the impact of RSV infection in a diverse hospitalized adult population that included HSCT, SOT, and non-transplant patients from 2009 to 2012 . The timing of RSV infection in each season did not differ between transplant and non-transplant patients. A dramatic and sustained increase was seen in the number of cases identified between the 2009 RSV season and the 
Logistic regression model for death in all inpatients $(n=174)$

\begin{tabular}{|c|c|c|}
\hline Parameter & $\begin{array}{l}\text { Odds ratio ( } 95 \% \\
\text { confidence interval) }\end{array}$ & $P$-value \\
\hline Gender $=$ male (vs. female) & $3.317(0.605,18.195)$ & 0.1674 \\
\hline Age $>60^{*}$ (vs. age $\leq 60$ ) & $8.413(1.429,49.524)$ & 0.0185 \\
\hline $\begin{array}{l}\text { Lymphocyte count (per } 100 \\
\text { unit increase) }\end{array}$ & $0.866(0.749,1.001)$ & 0.0512 \\
\hline $\begin{array}{l}\text { Stem cell transplant (vs. no } \\
\text { transplant) }\end{array}$ & $0.595(0.086,4.115)$ & 0.5988 \\
\hline $\begin{array}{l}\text { Solid organ transplant (vs. } \\
\text { no transplant) }\end{array}$ & $0.414(0.049,3.496)$ & 0.4178 \\
\hline $\begin{array}{l}\text { Chronic lung disease } \\
\text { (vs. absent) }\end{array}$ & $1.146(0.207,6.346)$ & 0.8760 \\
\hline Fever (vs. absent) & $1.298(0.292,5.773)$ & 0.7317 \\
\hline $\begin{array}{l}\text { No abnormal CT findings } \\
\text { (vs. } \geq 1 \text { finding) }\end{array}$ & $0.282(0.052,1.526)$ & 0.1417 \\
\hline $\begin{array}{l}\text { Lower respiratory infection } \\
\text { (vs. upper) }\end{array}$ & $2.160(0.192,24.314)$ & 0.5331 \\
\hline $\begin{array}{l}* \text { Significant } P<0.05 \text {. } \\
\text { CT, computed tomography. }\end{array}$ & & \\
\hline
\end{tabular}

Table 2

following seasons, which may be attributable to multiple factors. The improved sensitivity of the new testing platform, over that of viral culture and antigen-based assays, may have had an impact, as has been well described $(20,21)$. More significantly, however, our transition to a PCR platform allowed bundled testing for influenza and RSV, whereas previously, RSV testing required a separate specific order in a patient with an influenza-like illness, likely leading to increased screening for RSV in adults overall. Within the transplant cohorts, patients were typically infected several years after their transplant, reflecting the risk for acquisition of RSV as a community-acquired rather than nosocomial infection.

We did not identify independent risk factors for mortality from RSV infection among the transplant populations. Among the entire study population, only age $>60$ years was significantly associated with a risk of death from RSV infection. Among the individually analyzed cohorts of HSCT, SOT, and non-transplant patients, age $>60$ years increased the odds of mortality, but only approached significance in non-transplant recipients $(\mathrm{OR}=11.4 ; P=0.065)$. Analysis of other potential risk factors associated with mortality found decreased odds of death of $13.4 \%(\mathrm{OR}=0.866)$ per 100 cell increase in ALC among the entire study population, with this effect also approaching statistical significance $(P=0.051)$. When stratified by HSCT, SOT, and non- transplant cohorts, ALC was found to be a significant risk factor of death only in non-transplant patients $(\mathrm{OR}=0.092 ; P=0.045)$. A recent study of RSV in 181 HSCT recipients showed $24 \%$ of patients progressing to LRTI and identified risk factors for progression including smoking, high-dose total body irradiation, and ALC $\leq 100 \mathrm{~mm}^{3}$ at the onset of URTI symptoms (22). Many previous studies have commented on the increased risk of progressive infection and mortality in patients with lymphopenia (ALC $\leq 100 \mathrm{~mm}^{3}$ ) and older age (11, 22-25).

In this cohort, mortality was $6.9 \%(12 / 174$ patients). All 12 patients who died had evidence of LRTI at the time of admission. HSCT and SOT recipients did not have a statistically significant difference in mortality, when compared to non-transplant patients. In fact, HSCT recipients had a lower mortality (4.9\%) than either SOT recipients (10.9\%) or non-transplant patients (6.6\%). Although previous studies have not shown a significant attributable mortality to RSV infection in SOT recipients, recent studies of HSCT recipients with RSV LRTI have demonstrated mortality rates of $30-50 \%$ (9-13), and in elderly populations RSV infection may lead to 10,000 deaths annually (25). Several factors may have led to a relatively low mortality among the HSCT recipients in our study. HSCT patients were less likely to have LRTI at the time of admission than were other patients admitted with RSV infection, perhaps owing to clinical awareness of increased mortality risk from respiratory infections in this population, thereby leading to a lower threshold for admission for respiratory infection by healthcare providers, and to more frequent screening in the outpatient setting. The SOT and nontransplant cohorts may also have been at higher risk for mortality for reasons unrelated to transplantation status, such as age and chronic lung disease.

Mortality from RSV in the general population has been demonstrated to disproportionately affect the elderly population, with nearly $80 \%$ of deaths occurring in patients $>65$ years of age (25). Within the elderly population with RSV infection, chronic lung disease has been demonstrated as a risk factor for hospitalization (4). In this study, the non-transplant cohort had a mean age of 62 years, significantly older than the HSCT cohort. Likewise, both the SOT cohort and non-transplant cohort both had significantly higher prevalence of chronic lung disease, potentially contributing to the higher observed mortality rates.

Our study was unable to determine if aerosolized or oral ribavirin had an impact on the overall mortality of RSV in LRTI, or in the HSCT cohort alone. In the study, over half the HSCT patients received ribavirin therapy, compared with only $21 \%$ of SOT recipients and $9 \%$ of 
non-transplant patients. This difference is mostly a reflection of institutional policy, which restricts inhaled ribavirin to patients with a recent HSCT or with chronic graft-versus-host disease requiring immunosuppressive therapy. The efficacy of ribavirin for RSV infection remains controversial, although off-label use in HSCT and in heart and lung transplant recipients is common. The only randomized-controlled trial of ribavirin in HSCT recipients with RSV URTI was not able to definitively demonstrate efficacy, owing to low patient accrual (23). However, observational studies in HSCT recipients have shown oral and aerosolized ribavirin to be well tolerated, to prevent progression of URTI to LRTI, and to decrease mortality in LRTI $(9,13,26)$.

Several drugs are currently in the early stages of development for the treatment of RSV, but have not yet received U.S. Food and Drug Administration approval. Compounds are being developed that prevent RSV from fusing with host cell surfaces by targeting the RSV F protein. Other compounds inhibit the RSV polymerase or bind to the N-terminal region of nucleocapsid protein (27). Monoclonal antibodies have not been shown to have significant clinical activity when studied in children (28).

Our study has several limitations. As with most retrospective studies, incomplete or missing data may affect study statistics and outcome measures. Also, as testing for RSV is not mandatory for all adult patients with influenza-like illness at our institution, it is possible that some RSV infections in hospitalized patients were not detected, and therefore not included in the study. Similarly, as many patients transplanted at our institution receive acute care closer to their homes, some RSV infections in these populations may have been missed. Another important point is that no significant conclusions can be made about the treatment effects of oral or inhaled ribavirin for this hospitalized population, because these therapies were not administered in a consistent manner to any of the patient cohorts. Lack of a historical control group also limits assessment of efficacy of this treatment in the patients who received therapy during this study. Finally, the sample size of this study, while larger than many other descriptive studies of RSV infection in immunocompromised hosts, may have lacked the power necessary to identify certain risk factors for mortality.

In conclusion, this study did not identify any significant degree of increased mortality or morbidity associated with RSV infection in hospitalized adult HSCT or SOT recipients vs. non-transplant adult patients. We did demonstrate, however, that increased age and low ALCs are important risk factors for mortality in non-transplant patients, consistent with other prior studies. Although we cannot exclude the possibility of a beneficial treatment effect of ribavirin in the HSCT cohort, our results did not demonstrate decreased mortality in any specific patient cohort treated with ribavirin. Newer antiviral therapies for RSV are also in development and currently undergoing clinical trials in immunocompromised populations (29). Our results suggest that inclusion of certain non-transplant patients in future trials may be of benefit, with particular attention to patients with age $>60$, who are at high risk of complications from RSV infection.

\section{Acknowledgments:}

Conflicts of interest: There are no conflicts of interest.

\section{References}

1. Hall CB. Respiratory syncytial virus in young children. Lancet 2010; 375 (9725): 1500-1502.

2. Hall CB, Simoes EA, Anderson LJ. Clinical and epidemiologic features of 4 respiratory syncytial virus. Curr Top Microbiol Immunol 2013; 372 : 39-57.

3. Dowell SF, Anderson LJ, Gary HE Jr, et al. Respiratory syncytial virus is an important cause of community-acquired lower respiratory infection among hospitalized adults. J Infect Dis 1996; 174 (3): 456-462.

4. Falsey AR, Hennessey PA, Formica MA, Cox C, Walsh EE. Respiratory syncytial virus infection in elderly and high-risk adults. N Engl J Med 2005; 352 (17): 1749-1759.

5. Griffin MR, Coffey CS, Neuzil KM, Mitchel EF Jr, Wright PF, Edwards KM. Winter viruses: influenza- and respiratory syncytial virus-related morbidity in chronic lung disease. Arch Intern Med 2002; 162 (11): 1229-1236.

6. Zambon MC, Stockton JD, Clewley JP, Fleming DM. Contribution of influenza and respiratory syncytial virus to community cases of influenza-like illness: an observational study. Lancet 2001; 358 (9291): 1410-1416.

7. Walsh EE, Peterson DR, Falsey AR. Risk factors for severe respiratory syncytial virus infection in elderly persons. J Infect Dis 2004; 189 (2): 233-238.

8. Meissner HC. Selected populations at increased risk from respiratory syncytial virus infection. Pediatr Infect Dis J 2003; 22 (2 Suppl): S40-S44; discussion S44-S45.

9. Waghmare A, Campbell AP, Xie H, et al. Respiratory syncytial virus lower respiratory disease in hematopoietic cell transplant recipients: viral RNA detection in blood, antiviral treatment, and clinical outcomes. Clin Infect Dis 2013; 57 (12): 1731-1741.

10. Chemaly RF, Ghosh S, Bodey GP, et al. Respiratory viral infections in adults with hematologic malignancies and human stem cell transplantation recipients: a retrospective study at a major cancer center. Medicine (Baltimore) 2006; 85 (5): 278-287.

11. Ljungman $\mathrm{P}$, Ward $\mathrm{KN}$, Crooks BN, et al. Respiratory virus infections after stem cell transplantation: a prospective study from the infectious diseases working party of the European Group for Blood and Marrow Transplantation. Bone Marrow Transplant 2001; 28 (5): 479-484. 
Pilie et al: RSV outcomes in transplant vs. non-transplant patients

12. Avetisyan G, Mattsson J, Sparrelid E, Ljungman P. Respiratory syncytial virus infection in recipients of allogeneic stem-cell transplantation: a retrospective study of the incidence, clinical features, and outcome. Transplantation 2009; 88 (10): 1222-1226.

13. Shah JN, Chemaly RF. Management of RSV infections in adult recipients of hematopoietic stem cell transplantation. Blood 2011; 117 (10): 2755-2763.

14. Kim YJ, Boeckh M, Englund JA. Community respiratory virus infections in immunocompromised patients: hematopoietic stem cell and solid organ transplant recipients, and individuals with human immunodeficiency virus infection. Semin Respir Crit Care Med 2007; 28 (2): 222-242.

15. Khanna N, Widmer AF, Decker M, et al. Respiratory syncytial virus infection in patients with hematological diseases: singlecenter study and review of the literature. Clin Infect Dis 2008; 46 (3): 402-412.

16. Small TN, Casson A, Malak SF, et al. Respiratory syncytial virus infection following hematopoietic stem cell transplantation. Bone Marrow Transplant 2002; 29 (4): 321-327.

17. Lopez-Medrano F, Aguado JM, Lizasoain M, et al. Clinical implications of respiratory virus infections in solid organ transplant recipients: a prospective study. Transplantation 2007; 84 (7): 851-856.

18. Weigt SS, Gregson AL, Deng JC, Lynch JP 3rd, Belperio JA. Respiratory viral infections in hematopoietic stem cell and solid organ transplant recipients. Semin Respir Crit Care Med 2011; 32 (4): 471-493.

19. Hologic. Available at: http://www.hologic.com/package-inserts/ clinical-diagnostics-blood-screening/assays-and-tests/prodesse/ proflu/assay.

20. Freymuth F, Vabret A, Cuvillon-Nimal D, et al. Comparison of multiplex PCR assays and conventional techniques for the diagnostic of respiratory virus infections in children admitted to hospital with an acute respiratory illness. J Med Virol 2006; 78 (11): 1498-1504.
21. Kadmon G, Levy I, Mandelboim M, et al. Polymerase-chainreaction-based diagnosis of viral pulmonary infections in immunocompromised children. Acta Paediatr 2013; 102 (6): e263-e268.

22. Kim YJ, Guthrie KA, Waghmare A, et al. Respiratory syncytial virus in hematopoietic cell transplant recipients: factors determining progression to lower respiratory tract disease. J Infect Dis 2014; 209 (8): 1195-1204.

23. Boeckh M, Englund J, Li Y, et al. Randomized controlled multicenter trial of aerosolized ribavirin for respiratory syncytial virus upper respiratory tract infection in hematopoietic cell transplant recipients. Clin Infect Dis 2007; 44 (2): 245-249.

24. Ghosh S, Champlin RE, Englund J, et al. Respiratory syncytial virus upper respiratory tract illnesses in adult blood and marrow transplant recipients: combination therapy with aerosolized ribavirin and intravenous immunoglobulin. Bone Marrow Transplant 2000; 25 (7): 751-755.

25. Thompson WW, Shay DK, Weintraub E, et al. Mortality associated with influenza and respiratory syncytial virus in the United States. JAMA 2003; 289 (2): 179-186.

26. Shah DP, Ghantoji SS, Shah JN, et al. Impact of aerosolized ribavirin on mortality in 280 allogeneic haematopoietic stem cell transplant recipients with respiratory syncytial virus infections. J Antimicrob Chemother 2013; 68 (8): $1872-1880$

27. Simoes EA, DeVincenzo JP, Boeckh M, et al. Challenges and opportunities in developing respiratory syncytial virus therapeutics. J Infect Dis 2015; 211 (Suppl 1): S1-S20.

28. Ramilo O, Lagos R, Saez-Llorens X, et al. Motavizumab treatment of infants hospitalized with respiratory syncytial virus infection does not decrease viral load or severity of illness. Pediatr Infect Dis J 2014; 33 (7): 703-709.

29. DeVincenzo JP, Whitley RJ, Mackman RL, et al. Oral GS-5806 activity in a respiratory syncytial virus challenge study. N Engl J Med 2014; 371 (8): 711-722. 